\title{
ÉTALE TRIVIALITY OF FINITE VECTOR BUNDLES OVER COMPACT COMPLEX MANIFOLDS
}

\author{
INDRANIL BISWAS
}

\begin{abstract}
A vector bundle $E$ over a projective variety $M$ is called finite if it satisfies a nontrivial polynomial equation with nonnegative integral coefficients. Introducing finite bundles, Nori proved that $E$ is finite if and only if the pullback of $E$ to some finite étale covering of $M$ is trivializable [No1. The definition of finite bundles extends naturally to holomorphic vector bundles over compact complex manifolds. We prove that a holomorphic vector bundle over a compact complex manifold $M$ is finite if and only if the pullback of $E$ to some finite étale covering of $M$ is holomorphically trivializable. Therefore, $E$ is finite if and only if it admits a flat holomorphic connection with finite monodromy. In $\overline{\mathrm{BP}}$ this result was proved under the extra assumption that the compact complex manifold $M$ admits a Gauduchon astheno-Kähler metric.
\end{abstract}

\section{INTRODUCTION}

Given a vector bundle $E$ on a projective variety and a polynomial $P(x)=\sum_{i=0}^{N} a_{i} x^{i}$, where $a_{i}$ are nonnegative integers, define

$$
P(E):=\bigoplus_{i=0}^{N}\left(E^{\otimes i}\right)^{\oplus a_{i}},
$$

where $E^{\otimes 0}$ is the trivial line bundle. A vector bundle $E$ is called finite if there are two distinct polynomials $P_{1}$ and $P_{2}$ of the above type such that the two vector bundles $P_{1}(E)$ and $P_{2}(E)$ are isomorphic; finite bundles were introduced by Nori in [No1]. The above definition clearly makes sense if $E$ is a holomorphic vector bundle on a compact complex manifold.

There are several equivalent definitions of finiteness [No1, p. 35, Lemma 3.1]. The one that is most useful to work with is the following: A vector bundle $E$ is finite if and only if there are finitely many vector bundles $V_{1}, \cdots, V_{p}$ such that

$$
E^{\otimes j}=\bigoplus_{i=1}^{p} V_{i}^{\oplus j_{i}}
$$

for all $j \geq 1$, where $j_{i}$ are nonnegative integers.

Nori proved that a vector bundle $E$ on a complex projective variety $Z$ is finite if and only if the pullback of $E$ to some finite étale covering of $Z$ is trivializable [No1, [No2]. In [No1], [No2] the base field is allowed to be any algebraically closed field, although we have stated here for complex numbers. When $Z$ is smooth, a vector bundle $E$ pulls back

2010 Mathematics Subject Classification. 32L10, 53C55, $14 \mathrm{D} 21$.

Key words and phrases. Finite bundle, monodromy, stability, Hermitian-Einstein metric. 
to the trivial bundle under some finite étale covering of $Z$ if and only if $E$ admits a flat holomorphic connection with finite monodromy.

It is natural is ask whether the above characterization of finite vector bundles remains valid for holomorphic vector bundles over compact complex manifolds.

In [BHS] this characterization of finite bundles was proved for holomorphic vector bundles on compact Kähler manifolds. In $[\mathrm{BP}$ this characterization was proved for holomorphic vector bundles on compact complex manifolds that admit a Gauduchon asthenoKähler metric.

The following is proved here (Theorem 4.3 and Corollary 4.4):

Theorem 1.1. Let $E$ be a holomorphic vector bundle over a compact complex manifold $M$. Then $E$ is finite if and only if the pullback of $E$ to some finite étale covering of $M$ is holomorphically trivializable. Also, $E$ is finite if and only if it admits a flat holomorphic connection with finite monodromy.

Both [BHS] and [BP] used, very crucially, the numerically flat bundles introduced by J.-P. Demailly, T. Peternell and M. Schneider in [DPS]. Finite bundles are numerically flat. Theorem 1.18 of [DPS, p. 311] proves a characterization of numerically flat bundles on compact Kähler manifolds. This theorem of [DPS] was directly used in [BHS], and it was extended in [BP, p. 5, Theorem 3.2] to numerically flat bundles on compact complex manifolds admitting a Gauduchon astheno-Kähler metric, which was then used in an essential way. The proof of Theorem 1.1 avoids use of numerically flat bundles.

\section{HolOMORPHIC BUNDLES ON COMPACT COMPLEX MANIFOLDS}

2.1. Finite vector bundles. Let $M$ be a compact connected complex manifold. A holomorphic vector bundle $E$ over $M$ is called decomposable if there are holomorphic vector bundles $V$ and $W$ over $M$ of positive ranks such that $V \oplus W$ is holomorphically isomorphic to $E$. A holomorphic vector bundle is called indecomposable if it is not decomposable. Clearly, any holomorphic vector bundle can be expressed as a direct sum of indecomposable vector bundles. Atiyah proved the following uniqueness theorem for such decompositions.

Theorem 2.1 ([At, p. 315, Theorem 3]). Let $E$ be a holomorphic vector bundle over $M$ holomorphically isomorphic to both $\bigoplus_{i=1}^{m} V_{i}$ and $\bigoplus_{j=1}^{n} W_{j}$, where $V_{i}, 1 \leq i \leq m$, and $W_{j}, 1 \leq j \leq n$, are all indecomposable vector bundles. Then $m=n$, and there is a permutation $\sigma$ of $\{1, \cdots, m\}$ such that $V_{i}$ is holomorphically isomorphic to $W_{\sigma(i)}$ for all $1 \leq i \leq m$.

Definition 2.2. A holomorphic vector bundle $V$ will be called a component of $E$ if there is another holomorphic bundle $W$ such that $V \oplus W$ is isomorphic to $E$. If a component $V$ is also indecomposable, then it would be called an indecomposable component. 
A holomorphic vector bundle $E$ on $M$ is called finite if there are finitely many holomorphic vector bundles $V_{1}, \cdots, V_{p}$ such that

$$
E^{\otimes j}=\bigoplus_{i=1}^{p} V_{i}^{\oplus j_{i}}
$$

for all $j \geq 1$, where $j_{i}$ are nonnegative integers ([No1, p. 35, Definition], [No1, p. 35, Lemma 3.1(d)]), [No2, p. 80, Definition]. As mentioned in the introduction, $E$ is finite if and only if there are two distinct polynomials $P_{1}$ and $P_{2}$, whose coefficients are nonnegative integers, such that $P_{1}(E)$ is holomorphically isomorphic to $P_{2}(E)$ [No1, p. 35, Definition], [No1, p. 35, Lemma 3.1(a)].

Any vector bundle of the form $\bigoplus_{i=1}^{N} W_{i}^{\oplus n_{i}}$, where $n_{i}$ are nonnegative integers, will be called a direct sum of copies of $W_{1}, \cdots, W_{N}$.

We can assume that each $V_{i}$ in the above definition is a component (see Definition 2.2) of some $E^{\otimes m}$. If $V_{i}$ is a component of $E^{\otimes m}$, then $V^{\otimes k}$ is a component of $E^{\otimes k m}$ for all $k \geq 1$. Hence using Theorem 2.1 it follows that $V^{\otimes k}$ is a direct sum of copies of the indecomposable components (see Definition 2.2) of $\bigoplus_{i=1}^{p} V_{i}$. So $V_{1}, \cdots, V_{p}$ are finite bundles. Similarly, it follows from Theorem 2.1 that any component of a finite bundle is also a finite bundle [No1, p. 36, Lemma 3.2(2)], [No2, p. 80, Lemma 3.2(2)].

Hence we may assume that each $V_{i}$ in (2.1) is indecomposable.

If $E_{1}$ and $E_{2}$ are finite vector bundles, then both $E_{1} \oplus E_{2}$ and $E_{1} \otimes E_{2}$ are also finite [No1, p. 36, Lemma 3.2(1)], [No2, p. 80, Lemma 3.2(1)]. A holomorphic line bundle is finite if and only if it is of finite order [No1, p. 36, Lemma 3.2(3)], [No2, p. 80, Lemma $3.2(3)]$. If $E$ is finite then clearly the dual bundle $E^{*}$ is also finite.

2.2. Semistable sheaves. Let $d$ be the complex dimension of $M$. A Gauduchon metric on $M$ is a Hermitian structure $g$ on $M$ such that the associated positive $(1,1)$-form $\omega_{g}$ on $M$ satisfies the equation

$$
\partial \bar{\partial} \omega_{g}^{d-1}=0
$$

Gauduchon metric exists [Ga, p. 502, Théorème]. Fix a Gauduchon metric $g$ on $M$. This enables us to define the degree of a torsionfree coherent analytic sheaf $F$ on $M$ as follows: Fix a Hermitian structure on the determinant line bundle $\operatorname{det}(F) \longrightarrow M$ (see Ko2, Ch. V, $\S 6]$ for determinant bundle), and denote by $\mathcal{K}$ the curvature of the corresponding Chern connection; now define

$$
\operatorname{degree}(F)=\frac{1}{2 \pi \sqrt{-1}} \int_{M} \mathcal{K} \wedge \omega_{g}^{d-1} \in \mathbb{R}
$$

[LT, p. 43, Definition 1.4.1]. Define the slope of $F$

$$
\mu(F):=\frac{\operatorname{degree}(F)}{\operatorname{rank}(F)} \in \mathbb{R} .
$$

This allows us to define stability and semistability [LT, p. 44, Definition 1.4.3]. We recall that a torsionfree coherent analytic sheaf $F$ on $M$ is called polystable if it is a direct sum of stable sheaves of same slope; in particular, polystable sheaves are semistable. 
For a torsionfree coherent analytic sheaf $F$ on $M$, define $\mu_{\max }(F)$ to be the slope of the maximal semistable subsheaf of $F$. In other words, $\mu_{\max }(F)$ is the slope of the first term in the Harder-Narasimhan filtration of $F$.

A holomorphic vector bundle on $M$ is polystable if it admits a Hermitian-Einstein metric [LT, p. 55, Theorem 2.3.2] (see also [Lu, p. 12, Proposition 4], [Ko2, p. 177-178, Theorem 8.3] and [Ko1]). Moreover, A stable holomorphic vector bundle on $M$ admits a Hermitian-Einstein metric [LY, p. 563, Theorem 1], [LT, p. 61, Theorem 3.0.1]. If $H_{1}$ and $H_{2}$ are Hermitian-Einstein metrics on $E$ and $F$ respectively, then $H_{1} \otimes H_{2}$ is a HermitianEinstein metric on $E \otimes F$. This immediately implies that the tensor product of two stable vector bundles is polystable. Now it is straight-forward to deduce the following:

Corollary 2.3. If $E$ and $F$ are polystable vector bundles, then $E \otimes F$ is also polystable.

For the following proposition see [HL, p. 22, Proposition 1.5.2] (in [HL, Proposition 1.5.2] Gieseker (semi)stability is used, but the proof works for $\mu-$ (semi)stability; see [HL, p. 25, Theorem 1.6.6] for it), [Ko2, p. 175-176, Theorem 7.18], [BDL, p. 1034, Proposition 3.1], [BTT, p. 998, Proposition 2.1].

Proposition 2.4. Let $E$ be a semistable holomorphic vector bundle on $M$, and let

$$
0=F_{0} \subset F_{1} \subset \cdots \subset F_{b-1} \subset F_{b}=E
$$

be a filtration of reflexive subsheaves such that $F_{i} / F_{i-1}$ is stable with $\mu\left(F_{i} / F_{i-1}\right)=\mu(E)$ for all $1 \leq i \leq b$. Then the holomorphic isomorphism class of the graded object

$$
\bigoplus_{i=1}^{b} F_{i} / F_{i-1}
$$

is independent of the choice of the filtration.

Definition 2.5. The stable sheaves $F_{i} / F_{i-1}, 1 \leq i \leq b$, in Proposition 2.4 will be called the stable graded factors of $E$.

\section{Homomorphisms of Finite BUNDLES}

Lemma 3.1. Let $E$ be a finite vector bundle on $M$ and

$$
\phi \in H^{0}(M, E) \backslash\{0\}
$$

a nonzero holomorphic section. Then $\phi$ does not vanish at any point of $M$.

Proof. Assume that $\phi$ vanishes at a point $x_{0} \in M$. Then

$$
\phi^{\otimes j} \in H^{0}\left(M, E^{\otimes j}\right)
$$

vanishes at $x_{0}$ of order at least $j$.

Take any holomorphic vector bundles $A$ on $M$. For any nonzero holomorphic section $h \in H^{0}(M, A) \backslash\{0\}$, let $\operatorname{Ord}_{A}\left(h, x_{0}\right)$ denote the order of vanishing of $h$ at $x_{0}$. It can be shown that the set of all such integers

$$
\left\{\operatorname{Ord}_{A}\left(h, x_{0}\right)\right\}_{h \in H^{0}(M, A) \backslash\{0\}}
$$


is bounded above. Indeed, the space of holomorphic sections of $A$ vanishing at $x_{0}$ of order at least $k$ is a subspace of the finite dimensional vector space $H^{0}(M, A)$, and $H^{0}(M, A)$ is filtered by such subspaces.

Consider the decomposition of $E^{\otimes j}$ in (2.1); fix an isomorphism of $E^{\otimes j}$ with the direct sum. Take any $1 \leq k \leq p$ such that

(1) $j_{k}>0$, and

(2) the projection of the section $\phi^{\otimes j}$ to some component $V_{k}$ of $E^{\otimes j}$ (of the $j_{k}$ components) is nonzero.

Since $\phi^{\otimes j}$ vanishes at $x_{0}$ of order at least $j$, the projection of $\phi^{\otimes j}$ to any component of $E^{\otimes j}$ vanishes at $x_{0}$ of order at least $j$ (if the projection does not vanish identically). But this contradicts the above observation that the orders of vanishing, at $x_{0}$, of the nonzero sections of a given holomorphic vector bundle are bounded above. This proves that $\phi$ does not vanish at any point of $M$.

Proposition 3.2. Let $E$ and $F$ be finite holomorphic vector bundles on $M$, and let

$$
\Phi: E \longrightarrow F
$$

be an $\mathcal{O}_{M}$-linear homomorphism. Then the image $\Phi(E)$ is a subbundle of $F$.

Proof. Let $r$ be the rank of $\Phi(E)$. We assume that $r>0$, because the proposition is obvious for $r=0$. Consider the holomorphic vector bundle

$$
\operatorname{Hom}\left(\bigwedge^{r} E, \bigwedge^{r} F\right)=\left(\bigwedge^{r} F\right) \otimes\left(\bigwedge^{r} E\right)^{*}
$$

Since $F$ is a finite vector bundle, we know that $\otimes^{r} F$ is also finite. Therefore, the direct summand $\bigwedge^{r} F$ of $\bigotimes^{r} F$ is also finite. Similarly, $\left(\bigwedge^{r} E\right)^{*}$ is also finite. Consequently, $\operatorname{Hom}\left(\bigwedge^{r} E, \bigwedge^{r} F\right)$ is a finite bundle.

Let

$$
\widehat{\Phi}:=\bigwedge^{r} \Phi \in H^{0}\left(M, \operatorname{Hom}\left(\bigwedge^{r} E, \bigwedge^{r} F\right)\right)
$$

be the homomorphism of $r$-th exterior products corresponding to $\Phi$. Since the rank of $\Phi(E)$ is $r$, the rank of the subsheaf $\widehat{\Phi}\left(\bigwedge^{r} E\right) \subset \bigwedge^{r} F$ is one, and the section $\widehat{\Phi}$ vanishes exactly on the closed subset of $M$ over which $\Phi(E)$ fails to be a subbundle of $F$. But $\widehat{\Phi}$ does not vanish anywhere by Lemma 3.1. Consequently, $\Phi(E)$ is a subbundle of $F$.

Lemma 3.3. Let $E$ be a finite vector bundle on $M$. Then $E$ is semistable, and

$$
\text { degree }(E)=0 \text {. }
$$

Proof. Consider the decomposition in (2.1). We have

$$
\mu\left(\bigoplus_{i=1}^{p} V_{i}^{\oplus j_{i}}\right)=\sum_{i=1}^{p} \frac{j_{i} \cdot \operatorname{rank}\left(V_{i}\right)}{\sum_{i=1}^{p} j_{i} \cdot \operatorname{rank}\left(V_{i}\right)} \mu\left(V_{i}\right),
$$

in particular, $\operatorname{Min}\left\{\mu\left(V_{i}\right)\right\}_{i=1}^{p} \leq \mu\left(\bigoplus_{i=1}^{p} V_{i}^{\oplus j_{i}}\right) \leq \operatorname{Max}\left\{\mu\left(V_{i}\right)\right\}_{i=1}^{p}$. Hence from (2.1) it follows that $\left\{\mu\left(E^{\otimes j}\right)\right\}_{j=1}^{\infty}$ is bounded. On the other hand,

$$
\mu\left(E^{\otimes j}\right)=j \cdot \mu(E) .
$$


Therefore, we have $\mu(E)=0$. This implies that degree $(E)=0$.

If $E$ is not semistable, take any subsheaf $W \subset E$ such that $\mu(W)>\mu(E)=0$. So $\operatorname{det}(W)$ is a subsheaf of $\bigwedge^{s} E \subset \bigotimes^{s} E$, where $s$ is the rank of $W$. Hence the line bundle $(\operatorname{det}(W))^{\otimes j}$ is a subsheaf of $\bigotimes^{s \cdot j} E$. Now from (2.1) it follows that $(\operatorname{det}(W))^{\otimes j}$ is a subsheaf of some $V_{i}$ for some $i \in\{1, \cdots, p\}$ (the projection of $(\operatorname{det}(W))^{\otimes j}$ to one of the direct summands in (2.1) has to be nonzero). This implies that

$$
\mu\left((\operatorname{det}(W))^{\otimes j}\right) \leq \operatorname{Max}\left\{\mu_{\max }\left(V_{1}\right), \cdots, \mu_{\max }\left(V_{p}\right)\right\} .
$$

Consequently, the collection of real numbers $\left\{\mu\left((\operatorname{det}(W))^{\otimes j}\right)\right\}_{j=1}^{\infty}$ is bounded above.

On the other hand, we have $\mu\left((\operatorname{det}(W))^{\otimes j}\right)=j s \cdot \mu(W)$. Since $\mu(W)>0$, this contradicts the above observation that $\left\{\mu\left((\operatorname{det}(W))^{\otimes j}\right)\right\}_{j=1}^{\infty}$ is bounded above. Therefore, $E$ is semistable.

Proposition 3.4. Let $E$ be a finite vector bundle on $M$, and let $Q$ be a torsionfree quotient of $E$ of degree zero. Then $Q$ is locally free.

Proof. The open subset of $M$ over which $Q$ is locally free will be denoted by $U$. The complement $M \backslash U$ is a complex analytic subspace of $M$ of codimension at least two; this is because $Q$ is torsionfree.

Let $r$ be the rank of $Q$. The quotient map $E \longrightarrow Q$ produces an $\mathcal{O}_{M}$-linear homomorphism

$$
\varphi: \bigwedge^{r} E \longrightarrow \operatorname{det}(Q)
$$

Note that the restriction $\left.\varphi\right|_{U}:\left.\left.\left(\bigwedge^{r} E\right)\right|_{U} \longrightarrow(\operatorname{det}(Q))\right|_{U}$ is surjective, because we have $\left.(\operatorname{det}(Q))\right|_{U}=\bigwedge^{r}\left(\left.Q\right|_{U}\right)$.

We shall first show that the holomorphic line bundle $\operatorname{det}(Q)$ is of finite order.

The image $\varphi\left(\bigwedge^{r} E\right) \subset \operatorname{det}(Q)$ will be denoted by $L$, where $\varphi$ is the homomorphism in (3.1). Hence $L^{j}$ is a quotient of $\left(\bigwedge^{r} E\right)^{\otimes j}$. Since $\left(\bigwedge^{r} E\right)^{\otimes j}$ is a component of $E^{\otimes r j}$ (see Definition 2.2), from Theorem 2.1 and (2.1) we conclude that $\left(\bigwedge^{r} E\right)^{\otimes j}$ is a direct sum of copies of $V_{1}, \cdots, V_{p}$ (recall that $V_{1}, \cdots, V_{p}$ are assumed to be indecomposable). The vector bundles $V_{1}, \cdots, V_{p}$ are semistable of degree zero by Lemma 3.3, and hence using Proposition 2.4 it follows that the stable quotients of degree zero of a direct sum of copies of $V_{1}, \cdots, V_{p}$ are just the stable quotients of degree zero of some $V_{i}$ occurring in the direct sum $\bigoplus_{i=1}^{p} V_{i}$.

Since degree $\left(L^{j} /\right.$ Torsion $)=j \cdot \operatorname{degree}(\operatorname{det}(Q))=0$, and $L^{j} /$ Torsion is a quotient of a direct sum of copies of $V_{1}, \cdots, V_{p}$, we conclude that $L^{j} /$ Torsion is a quotient of some $V_{i}$, $1 \leq i \leq p$. Each $V_{i}$ has only finitely many torsionfree quotients of rank one and degree zero (see Proposition 2.4); this can also be deduced from [BDL, p. 1034, Proposition 3.1] (this proposition says that the semistable vector bundle $V_{i}^{*}$ admits only finitely many isomorphisms classes of reflexive stable subsheaves of degree zero). Consequently, we have $\left.L^{\otimes a}\right|_{U}=\left.L^{\otimes b}\right|_{U}$ for some $a \neq b$. This implies that $\operatorname{det}(Q)^{\otimes a}=\operatorname{det}(Q)^{\otimes b}$, because the complement $M \backslash U$ is a complex analytic subspace of $M$ of codimension at least two, and $\left.L\right|_{U}=\left.\operatorname{det}(Q)\right|_{U}$. Therefore, the line bundle $\operatorname{det}(Q)$ is of finite order. 
Since $\operatorname{det}(Q)$ is a finite bundle, from Proposition 3.2 we conclude that the homomorphism $\varphi$ in (3.1) is surjective.

Consider the projective bundle $\mathbb{P}\left(\bigwedge^{r} E\right) \longrightarrow M$ parametrizing the hyperplanes in the fibers of $\bigwedge^{r} E$. Let $\operatorname{Gr}(E)$ be the Grassmann bundle over $M$ parametrizing the $r-$ dimensional quotients of the fibers of $E$. Let

$$
\beta: \operatorname{Gr}(E) \longrightarrow \mathbb{P}\left(\bigwedge^{r} E\right)
$$

be the Plücker embedding that sends a quotient $\widehat{q}_{x}: E_{x} \longrightarrow \mathcal{Q}_{x}$ of dimension $r$ to the kernel of the homomorphism $\bigwedge^{r} \widehat{q}_{x}: \bigwedge^{r} E_{x} \longrightarrow \bigwedge^{r} \mathcal{Q}_{x}$.

Let

$$
\sigma: M \longrightarrow \mathbb{P}\left(\bigwedge^{r} E\right)
$$

be the holomorphic section defined by the surjective homomorphism $\varphi$ in (3.1). The image of the restriction $\left.\sigma\right|_{U}$ lies in $\beta\left(\left.\operatorname{Gr}(E)\right|_{U}\right) \subset \mathbb{P}\left(\bigwedge^{r} E\right)$, where $\beta$ is the map in (3.2). Consequently, $\sigma(M)$ lies in $\beta(\operatorname{Gr}(E))$, because $\beta(\operatorname{Gr}(E))$ is a closed submanifold of $\mathbb{P}\left(\bigwedge^{r} E\right)$.

Since $\sigma(M)$ lies in $\beta(\operatorname{Gr}(E))$, it is evident that $Q$ is a quotient bundle of $E$.

\section{Flat Connection on finite Bundles}

A holomorphic vector bundle $E$ over $M$ would be called étale trivial if there is a finite connected étale covering

$$
\varpi: Y \longrightarrow M
$$

such that the vector bundle $\varpi^{*} E$ is holomorphically trivializable.

Proposition 4.1. Let $E$ be a finite stable vector bundle on $M$. Then $E$ is étale trivial.

Proof. Consider the vector bundles $V_{1}, \cdots, V_{p}$ in (2.1). As before, we assume that each $V_{i}$ is indecomposable and it is a component of some $E^{\otimes j}$. From the given condition that $E$ is stable it follows that $E^{\otimes j}$ is polystable (see Corollary 2.3). Using Lemma 3.3 we conclude that the degree of $E^{\otimes j}$ is zero. Hence any component (see Definition 2.2) of $E^{\otimes j}$ is also polystable of degree zero. In particular, all $V_{i}$ are polystable of degree zero. Since each $V_{i}$ is also indecomposable, it is stable of degree zero.

If $V_{i}$ is a component (see Definition 2.2) of $E^{\otimes j}$, then $V_{i}^{\otimes n}$ is a component of $E^{\otimes n j}$, so $V_{i}^{\otimes n}$ is also a direct sum of copies of $V_{1}, \cdots, V_{p}$.

Any nonzero homomorphism between two stable vector bundles of degree zero is an isomorphism. Take any reflexive subsheaf $S$ of degree zero of a direct sum $\mathcal{U}$ of copies of $V_{1}, \cdots, V_{p}$. Then $S$ is a subbundle of $\mathcal{U}$ by Proposition 3.4. Moreover, $S$ is a component of $\mathcal{U}$, because $\mathcal{U}$ is polystable of degree zero, and hence $S$ is a direct sum of copies of $V_{1}, \cdots, V_{p}$. Furthermore, any torsionfree quotient of degree zero of such a subsheaf $S$ is again a direct sum of copies of $V_{1}, \cdots, V_{p}$, because $S$ is polystable of degree zero. In 
particular, for any any homomorphism

$$
f: \bigoplus_{i=1}^{p} V_{i}^{\oplus c_{i}} \longrightarrow \bigoplus_{i=1}^{p} V_{i}^{\oplus d_{i}}
$$

both $\operatorname{kernel}(f)$ and cokernel $(f)$ are direct sums of copies of $V_{1}, \cdots, V_{p}$.

Fix a point $x_{0} \in M$. Assign the vector space $F_{x_{0}}$ to any holomorphic vector bundle $F$ on $M$. In view of the above observations, $V_{1}, \cdots, V_{p}$ produce a Tannakian category (defined in [No1, p. 30], [No2, p. 76]). Now using [No1, p. 31, Theorem 1.1], [No2, p. 77, Theorem 1.1], this Tannakian category produces a complex affine group-scheme. The isomorphism classes of indecomposable objects of this Tannakian category are contained in the union of the following three:

(1) $\left\{V_{1}, \cdots, V_{p}\right\}$,

(2) $\left\{V_{1}^{*}, \cdots, V_{p}^{*}\right\}$, and

(3) all indecomposable components (see Definition 2.2) of all $V_{i} \otimes V_{j}^{*}, 1 \leq i, j \leq p$. Note that $V_{i} \otimes V_{j}^{*}$ is polystable by Corollary 2.3, also, degree $\left(V_{i} \otimes V_{j}^{*}\right)=0$, because $\operatorname{degree}\left(V_{i}\right)=0$ for all $i$. So all indecomposable components of $V_{i} \otimes V_{j}^{*}$ are stable of degree zero.

Since the above union is a finite collection, from [No1, p. 31, Theorem 1.2], [No2, p. 77, Theorem 1.2] it follows that the group-scheme defined by the above Tannakian category is finite; this finite group will be denoted by $\Gamma$.

Fix a basis of $E_{x_{0}}$. Now the structure group $\operatorname{GL}\left(r_{E}, \mathbb{C}\right)$ of $E$, where $r_{E}$ denotes the rank of $E$, has a reduction of structure group to this group $\Gamma$ [No2, p. 79, Theorem 2.9] (see also [No2, p. 34, Proposition 2.9]). Since our group $\Gamma$ is finite, the proof of [No2, Theorem 2.9] remains valid without any modification.

Therefore, there is a finite étale Galois covering $\varpi: \widehat{Y} \longrightarrow M$ with Galois group $\Gamma$ such that the vector bundle $\varpi^{*} E$ is holomorphically trivializable. Now taking $Y$ to be a connected component of $\widehat{Y}$ we conclude that $E$ is étale trivial.

Let $E$ be a finite vector bundle over $M$. As in Proposition 2.4, let

$$
0=F_{0} \subset F_{1} \subset \cdots \subset F_{m-1} \subset F_{m}=E
$$

be a filtration of reflexive subsheaves of $E$ such that $F_{k} / F_{k-1}$ is stable of degree zero for all $1 \leq k \leq m$. From Proposition 3.4 we know that each $F_{k}$ is a subbundle of $E$.

Lemma 4.2. For every $1 \leq k \leq m$, the holomorphic vector bundle $F_{k} / F_{k-1}$ in (4.1) is finite.

Proof. Consider the vector bundles $V_{1}, \cdots, V_{p}$ in (2.1). Since they are all finite, just as in (4.1), for every $1 \leq i \leq p$ there is a filtration of holomorphic subbundles

$$
0=U_{0}^{i} \subset U_{1}^{i} \subset \cdots \subset U_{n_{i}-1}^{i} \subset U_{n_{i}}^{i}=V_{i}
$$


such that $U_{\nu}^{i} / U_{\nu-1}^{i}$ is a stable vector bundle of degree zero for all $1 \leq \nu \leq n_{i}$. Now consider the collection of stable vector bundles

$$
\left\{\left\{U_{\nu}^{i} / U_{\nu-1}^{i}\right\}_{\nu=1}^{n_{i}}\right\}_{i=1}^{p}
$$

From Proposition 2.4 it follows that this collection does not depend on the choice of the filtrations in (4.2).

Consider the vector bundle $F_{k} / F_{k-1}$ in the statement of the proposition. Since $F_{k} / F_{k-1}$ is stable, by Corollary 2.3 , the vector bundle $\left(F_{k} / F_{k-1}\right)^{\otimes j}$ is polystable for every positive integer $j$. Also,

$$
\mu\left(\left(F_{k} / F_{k-1}\right)^{\otimes j}\right)=j \cdot \mu\left(F_{k} / F_{k-1}\right)=0 .
$$

Since $F_{k}^{\otimes j}$ is a subbundle of $E^{\otimes j}$ of degree zero, and $E^{\otimes j}$ is semistable of degree zero (recall that $E^{\otimes j}$ is a finite bundle), it follows that $F_{k}^{\otimes j}$ is semistable of degree zero. Also, $\left(F_{k} / F_{k-1}\right)^{\otimes j}$ is a polystable quotient of $F_{k}^{\otimes j}$ of degree zero. From these it follows that $\left(F_{k} / F_{k-1}\right)^{\otimes j}$ is a direct sum of copies of stable graded factors of $E^{\otimes j}$ (see Definition 2.5). Now using (2.1) and Proposition 2.4 we conclude that $\left(F_{k} / F_{k-1}\right)^{\otimes j}$ is a direct sum of copies of the vector bundles in the collection in (4.3). Therefore, $F_{k} / F_{k-1}$ is a finite bundle.

Theorem 4.3. A holomorphic vector bundle on $M$ is finite if and only if it is étale trivial.

Proof. Let $E$ be a finite vector bundle on $M$. Take a filtration of subbundles of it

$$
0=F_{0} \subset F_{1} \subset \cdots \subset F_{m-1} \subset F_{m}=E
$$

as in (4.1). From Lemma 4.2 and Proposition 4.1 we know that $F_{k} / F_{k-1}$ is étale trivial for all $1 \leq k \leq m$. Hence there is a finite connected étale Galois covering

$$
\varpi: Y \longrightarrow M
$$

such that for the pulled back filtration

$$
0=\varpi^{*} F_{0} \subset \varpi^{*} F_{1} \subset \cdots \subset \varpi^{*} F_{m-1} \subset \varpi^{*} F_{m}=\varpi^{*} E,
$$

the quotient bundle $\left(\varpi^{*} F_{k}\right) /\left(\varpi^{*} F_{k-1}\right)=\varpi^{*}\left(F_{k} / F_{k-1}\right)$ is trivializable for all $1 \leq k \leq m$ (take a connected component of the fiber product of the étale Galois coverings for each $\left.F_{k} / F_{k-1}\right)$. Also, $\varpi^{*} E$ is finite because $E$ is so.

Lemma 4.3 of $[\mathrm{BP}]$ says that if a finite vector bundle $W$ admits a filtration of holomorphic subbundles such that each successive quotient is holomorphically trivializable, then $W$ is holomorphically trivializable. From this and (4.4) it follows that $\varpi^{*} E$ is holomorphically trivializable. Hence $E$ is étale trivial.

To prove the converse, let $E$ be an étale trivial vector bundle on $M$. Let

$$
\varpi: Y \longrightarrow M
$$

be a connected étale covering such that $\varpi^{*} E$ is holomorphically trivializable. Consider the trivial connection $\nabla$ on $\varpi^{*} E$ corresponding to any holomorphic trivialization of $\varpi^{*} E$. This connection $\nabla$ does not depend on the choice of the holomorphic trivialization of $\varpi^{*} E$. 
It can be shown that $\nabla$ descends to a connection on $E$. Indeed, by taking an étale covering of $Y$ we may assume that $\varpi$ is Galois. The connection $\nabla$ is invariant under the action of the Galois group $\operatorname{Gal}(\varpi)$ on $\varpi^{*} E$, and hence $\nabla$ descends to a connection on $E$. This descended connection on $E$ is holomorphic flat because $\nabla$ is holomorphic flat; also, its monodromy group is finite because $\varphi$ is a finite covering and the monodromy group of $\nabla$ is trivial. This immediately implies that $E$ is finite; see [BP, p. 7, Section 4].

Theorem 4.3 and its proof together have the following corollary.

Corollary 4.4. A holomorphic vector bundle on $M$ is finite if and only if it admits a flat holomorphic connection with finite monodromy.

\section{ACKNOWLEDGEMENTS}

The author is grateful to Madhav Nori and Vamsi Pingali for helpful comments. He is partially supported by a J. C. Bose Fellowship.

\section{REFERENCES}

[At] M. F. Atiyah, On the Krull-Schmidt theorem with application to sheaves, Bull. Soc. Math. Fr. 84 (1956), 307-317.

[BDL] I. Biswas, S. Dumitrescu and M. Lehn, On the stability of flat complex vector bundles over parallelizable manifolds, Com. Ren. Math. Acad. Sci. Paris 356 (2018), 1030-1035.

[BHS] I. Biswas, Y. Holla, and G. Schumacher, On a characterization of finite vector bundles as vector bundles admitting a flat connection with finite monodromy group, Proc. Amer. Math. Soc. 128 (2000), 3661-3669.

[BP] I. Biswas and V. P. Pingali, A characterization of finite vector bundles on Gauduchon asthenoKähler manifolds, Épijournal Geom. Algébr. 2 (2018), Art. 6.

[BTT] N. Buchdahl, A. Teleman and M, Toma, A continuity theorem for families of sheaves on complex surfaces, J. Topol. 10 (2017), 995-1028.

[DPS] J.P. Demailly, T. Peternell, and M. Schneider, Compact complex manifolds with numerically effective tangent bundles, Jour. Algebraic Geom. 3 (1994), 295-346.

[Ga] P. Gauduchon, La 1-forme de torsion d'une variété hermitienne compacte, Math. Ann. 267 (1984), $495-518$.

[HL] D. Huybrechts and M. Lehn, The geometry of moduli spaces of sheaves, Aspects of Mathematics, E31. Friedr. Vieweg \& Sohn, Braunschweig, 1997.

[Ko1] S. Kobayashi, Curvature and stability of vector bundles, Proc. Japan Acad. Ser. A Math. Sci. 58 (1982), 158-162.

[Ko2] S. Kobayashi, Differential geometry of complex vector bundles, Publications of the Mathematical Society of Japan, 15. Kanô Memorial Lectures, 5, Princeton University Press, Princeton, NJ; Princeton University Press, Princeton, NJ, 1987.

[LY] J. Li and S.-T. Yau, Hermitian-Yang-Mills connection on non-Kähler manifolds, Mathematical aspects of string theory (San Diego, Calif., 1986), 560-573, Adv. Ser. Math. Phys., 1, World Sci. Publishing, Singapore, 1987.

[Lu] M. Lübke, Stability of Einstein-Hermitian vector bundles, Manuscripta Math. 42 (1983), 245-257.

[LT] M. Lübke and A. Teleman, The Kobayashi-Hitchin correspondence, World Scientific Publishing Co., Inc., River Edge, NJ, 1995.

[No1] M. V. Nori, On the representations of the fundamental group scheme, Compos. Math. 33 (1976), $29-41$.

[No2] M. V. Nori, The fundamental group-scheme, Proc. Ind. Acad. Sci. (Math. Sci.) 91 (1982), 73122. 
School of Mathematics, Tata Institute of Fundamental Research, Homi Bhabha Road, Mumbai 400005, INDiA

E-mail address: indranil@math.tifr.res.in 